\title{
ON ELEMENTARY GROUPS
}

\author{
ERNEST L. STITZINGER
}

ABstract. Bechtell has defined a group $G$ to be elementary if the Frattini subgroup of each subgroup of $G$ is the identity. In this note we prove the following: If the derived group of $G$ is nilpotent, then necessary and sufficient conditions that $G$ be elementary are that the Frattini subgroup of $G$ be the identity and that the Frattini subgroup of some Carter subgroup $K$ of $G$ be equal to the derived group of $K$.

Bechtell in [1] has defined a group $G$ to be elementary if the Frattini subgroup of each subgroup $H$ of $G, \operatorname{Fr}(H)$, is the identity. In this note we find that if $G^{1}$ is nilpotent then necessary and sufficient conditions that $G$ be elementary are that $\operatorname{Fr}(G)=1$ and $\operatorname{Fr}(K)=K^{1}$ for some (hence all) Carter subgroup $K$ of $G$. Only finite solvable groups are considered here and the notation is as in [4].

LemMa 1. Let $A$ be an invariant subgroup of $G$ and $B$ be a subgroup of $G$ such that $A \subseteq B$ and $B / A$ is a Carter subgroup of $G / A$. Let $H$ be $a$ Carter subgroup of $B$. Then $H$ is a Carter subgroup of $G$.

Proof. $H$ is nilpotent. Let $x \in N_{G}(H)$. Then $x A \in N_{G / A}(H A / A)$. By a remark in [2], $H A / A$ is a Carter subgroup of $B / A$ and $B / A$ is nilpotent. Hence $H A=B$ and $x A \in N_{G / A}(B / A)$. Therefore $x \in B$ and hence $x \in N_{B}(H)=H$.

Lemma 2. Let $G^{1}$ be complemented in $G$ by a subgroup $K$ such that $G^{1}$ and $K$ are abelian and $G^{1} \cap Z(G)=1$. Then Carter subgroups of $G$ are precisely those subgroups of $G$ which are complements to $G^{1}$.

Proof. $K$ is nilpotent. If $N_{G}(K)$ contains $K$ properly then there exists $x \in N_{G}(K) \cap G^{1}, x \neq 1$. Then for all $k \in K, x k x^{-1} \in K$ which yields $x k x^{-1} k^{-1} \in K \cap G^{1}=1$. Therefore $x \in C_{G}(K)$ and, since $G^{1}$ is abelian, $x \in Z(G)$, whence $x=1$, a contradiction. Therefore $N_{G}(K)$ $=K$ and $K$ is a Carter subgroup of $G$. If $J$ is another Carter subgroup of $G$, then $K$ and $J$ are conjugate, hence $J$ also complements $G^{1}$.

Corollary. Let $G^{1}$ be nilpotent and $\operatorname{Fr}(G)=1$. Then Carter subgroups of $G$ are precisely those subgroups of $G$ which complement $G^{1}$.

Proof. By Satz 12 in [3], Fit $(G)=\operatorname{Soc}(G)$, hence $G^{1}$ is abelian. By

Received by the editors February 5, 1970.

AMS 1969 subject classifications. Primary 2040, 2025.

Key words and phrases. Elementary group, Carter subgroup, Frattini subgroup. 
Satz 7 in [3], there exists a complement $K$ to $G^{1}$ in $G$. Since $[K, K]$ $\subseteq K \cap G^{1}=1, K$ is abelian. Furthermore $Z(G) \cap G^{1} \subseteq \operatorname{Fr}(G)=1$. Hence $G$ satisfies the hypothesis of Lemma 2.

Lemma 3. Let $G^{1}$ be nilpotent. Then the following are equivalent:

(1) $\operatorname{Fr}(G)=1$.

(2) $\operatorname{Fit}(G)=\operatorname{Soc}(G)$ and $\operatorname{Fit}(G)$ is complemented by a subgroup and $\operatorname{Fr}(G) \subseteq G^{1}$.

(3) $G^{1}$ is abelian, is completely reducible under inner automorphisms of $G$, is complemented by a subgroup and $\operatorname{Fr}(G) \subseteq G^{1}$.

Proof. That (1) implies (2) is well known even if $G^{1}$ is not nilpotent.

Assume (2) holds and proceed by induction on the order of $G$. Since $G^{1} \subseteq \operatorname{Fit}(G)=\operatorname{Soc}(G), G^{1}$ is abelian. If every minimal invariant subgroup of $G$ is contained in $G^{1}$, then $G^{1}=\operatorname{Soc}(G)$ and (3) follows. Therefore let $A$ be a minimal invariant subgroup of $G$ such that $A \nsubseteq G^{1}$. Hence $A \nsubseteq \operatorname{Fr}(G)$ and $A$ is complemented by a maximal subgroup, say $K$. Since $[G, A] \subseteq G^{1} \cap A=1, A$ is central in $G$, hence $G$ is the direct product of $A$ and $K . K$ inherits the conditions (2), hence $K$ satisfies (3) by induction. It now follows that $G$ also satisfies (3).

Assume (3) holds. Then $G^{1}$ is the direct product of minimal invariant subgroups of $G$ which we denote by $A_{1}, \cdots, A_{8}$ and $G$ is the semidirect product of $G^{1}$ and a subgroup, say $K$. Now $K_{i}=K A_{1} \cdots \hat{A}_{i} \cdots A_{s}$ is a maximal subgroup of $G$ since if $M$ is a maximal subgroup of $G$ properly containing $K_{i}$, then $M \cap A_{i} \neq 1$ and $M \cap A_{i}$ is invariant in $G$, hence equals $A_{i}$. This implies that $M=G$, a contradiction. Therefore $\operatorname{Fr}(G) \subseteq K$ and $\operatorname{Fr}(G) \subseteq K \cap G^{1}=1$. Hence (1) holds.

Lemma 4. Let $G^{1} \subseteq \operatorname{Soc}(G), A$ be an invariant subgroup of $G$ contained in $G^{1}$ and $Z(G) \cap G^{1}=1$. Then $Z(G / A) \cap G^{1} / A$ is the identity of $G / A$.

Proof. $G^{1}$ is the direct product of $A$ and an invariant subgroup of $G$, say $B$. Let $x A \in Z(G / A) \cap G^{1} / A$. Since $x A \in Z(G / A), x g x^{-1} g^{-1} \in A$ for all $g \in G$. Since $x A \in G^{1} / A, x=a b$ where $a \in A$ and $b \in B$. Then $x g x^{-1} g^{-1}=a b g b^{-1} a^{-1} g^{-1}=a c b g b^{-1} g^{-1}$ where $c=g a^{-1} g^{-1} \in A$. Hence $b g b^{-1} g^{-1} \in A \cap B=1$ for all $g \in G$ and therefore $b \in Z(G) \cap G^{1}=1$. Consequently $x \in A$ and $Z(G / A) \cap G^{1} / A$ is the identity of $A$.

THEOREM. Let $G$ be a group such that $G^{1}$ is nilpotent. Then $G$ is elementary if and only if $\operatorname{Fr}(G)=1$ and $\operatorname{Fr}(K)=K^{1}$ for some Carter subgroup $K$ of $G$. 
Proof. If $G$ is elementary, then $\operatorname{Fr}(G)=1$. Let $K$ be a Carter subgroup of $G$. Then $K$ complements $G^{1}$, hence $K$ is abelian. Therefore $K^{1}=1=\operatorname{Fr}(K)$.

Conversely, in order to show a contradiction, let $G$ be a group of minimal order such that $G^{1}$ is nilpotent, $\operatorname{Fr}(G)=1, \operatorname{Fr}(K)=K^{1}$ for some Carter subgroup $K$ of $G$ and $G$ is not elementary. Then $\operatorname{Fr}(J)$ $=J^{1}$ for every Carter subgroup $J$ of $G$. Let $M$ be a subgroup of $G$. If $M \supseteq G^{1}$, then $M$ is invariant in $G$ and $\operatorname{Fr}(M) \subseteq \operatorname{Fr}(G)=1$. Assume that $G^{1} \Phi M$ and that $M G^{1} \subset G$. Since $G^{1} \subseteq M G^{1}, \operatorname{Fr}\left(M G^{1}\right)=1$ and $G / M G^{1}$ is abelian, hence $G / M G^{1}$ is its own Carter subgroup. Then, if $H$ is a Carter subgroup of $M G^{1}, H$ is also a Carter subgroup of $G$ by Lemma 1 , hence by assumption $\operatorname{Fr}(H)=H^{1}$. By the minimality of $G, M G^{1}$ is elementary, hence $\operatorname{Fr}(M)=1$.

Suppose now that $M G^{1}=G$. Since $G^{1}$ is abelian, $G^{1} \cap M$ is an invariant subgroup in $G$ and $M /\left(G^{1} \cap M\right)$ complements $G^{1} /\left(G^{1} \cap M\right)$ $=\left(G /\left(G^{1} \cap M\right)\right)^{1}$ in $G /\left(G^{1} \cap M\right)$. Since $\operatorname{Fr}(G)=1, G^{1} \cap Z(G)=1$ and, by Lemma $3, G^{1} \subseteq \operatorname{Soc}(G)$. Therefore, by Lemma $4,\left(G /\left(G^{1} \cap M\right)\right)^{1}$ $\cap Z\left(G /\left(G^{1} \cap M\right)\right)$ is the identity of $G /\left(G^{1} \cap M\right)$. Hence $M /\left(G^{1} \cap M\right)$ is a Carter subgroup of $G /\left(G^{1} \cap M\right)$ by Lemma 2. Let $K$ be a Carter subgroup of $M$. By Lemma $1, K$ is a Carter subgroup of $G$. Hence $K$ is a complement to $G^{1}$ and $K\left(G^{1} \cap M\right)=M$ since $K \subseteq M$. Therefore, $K$ is a complement to $G^{1} \cap M$ in $M$. $G^{1} \cap M$ is completely reducible under the inner automorphisms of $M$ and $M^{1} \subseteq G^{1} \cap M$, hence $M^{1}$ is completely reducible under the inner automorphisms of $M$. Let $J$ be a complement to $M^{1}$ in $G^{1} \cap M$ which is invariant under the inner automorphisms of $M$. Then $J \subseteq Z(M) \cap G^{1}$, hence $G^{1} \cap M=M^{1} J$ $\subseteq M^{1}\left(G^{1} \cap Z(M)\right) \subseteq G^{1} \cap M$. Therefore $G^{1} \cap M=M^{1}\left(G^{1} \cap Z(M)\right)$. But $Z(M) \subseteq N_{M}(K)=K$, hence $G^{1} \cap Z(M) \subseteq G^{1} \cap K=1$ and $G^{1} \cap M=M^{1}$. Furthermore, $\operatorname{Fr}(K)=K^{1}$ as a Carter subgroup of $G$. Now $M$ satisfies part (3) of Lemma 3, hence $\operatorname{Fr}(M)=1$. Consequently, $\operatorname{Fr}(M)=1$ for every subgroup $M$ of $G$ and $G$ is elementary, a contradiction. This completes the proof.

\section{REFERENCES}

1. H. Bechtell, Elementary groups, Trans. Amer. Math. Soc. 114 (1965), 355-362. MR 31 \#243.

2. R. Carter, Nilpotent self-normalizing subgroups of soluble groups, Math. Z. 75 (1960/61), 136-139. MR 23 \#A928.

3. W. Gaschütz, Über die $\phi$-Untergruppe endlicher Gruppen, Math. Z. 58 (1953), 160-170. MR 15, 285.

4. W. Scott, Group theory, Prentice-Hall, Englewood Cliffs, N. J., 1964. MR 29 \#4785.

North Carolina State University, Raleigh, North Carolina 27607 